Article

\title{
Visualization of Escher-like Spiral Patterns in Hyperbolic Space
}

\author{
Chongyang Qiu ${ }^{1}\left(\mathbb{D}\right.$, Xinfei Li $^{2}$, Jianhua Pang ${ }^{2}$ and Peichang Ouyang $2,3, * \mathbb{C}$ \\ 1 Human Sciences Department, Gannan University of Science and Technology, Ganzhou 341000, China; \\ qiucy80@126.com \\ 2 School of Science, Guangxi University of Science and Technology, Liuzhou 545006, China; \\ xfli@gxust.edu.cn (X.L.); 100001019@gxust.edu.cn (J.P.) \\ 3 School of Mathematics and Physics, Jinggangshan University, Ji'an 343009, China \\ * Correspondence: g_fcayang@163.com
}

check for

updates

Citation: Qiu, C.; Li, X.; Pang, J.;

Ouyang, P. Visualization of

Escher-like Spiral Patterns in

Hyperbolic Space. Symmetry 2022, 14,

134. https://doi.org/10.3390/

sym14010134

Academic Editor: Alexei Kanel-Belov

Received: 18 November 2021

Accepted: 3 January 2022

Published: 11 January 2022

Publisher's Note: MDPI stays neutral with regard to jurisdictional claims in published maps and institutional affiliations.

Copyright: (C) 2022 by the authors Licensee MDPI, Basel, Switzerland. This article is an open access article distributed under the terms and conditions of the Creative Commons Attribution (CC BY) license (https:// creativecommons.org/licenses/by/ $4.0 /)$.

\begin{abstract}
Spirals, tilings, and hyperbolic geometry are important mathematical topics with outstanding aesthetic elements. Nonetheless, research on their aesthetic visualization is extremely limited. In this paper, we give a simple method for creating Escher-like hyperbolic spiral patterns. To this end, we first present a fast algorithm to construct Euclidean spiral tilings with cyclic symmetry. Then, based on a one-to-one mapping between Euclidean and hyperbolic spaces, we establish two simple approaches for constructing spiral tilings in hyperbolic models. Finally, we use wallpaper templates to render such tilings, which results in the desired Escher-like hyperbolic spiral patterns. The method proposed is able to generate a great variety of visually appealing patterns.
\end{abstract}

Keywords: spiral; tiling; hyperbolic geometry; visualization; Escher

\section{Introduction}

Spirals are sometimes called the "curve of nature" since they make up the general laws of animals, plant growth [1], and the universe at large. In nature, for both plants and animals, their appearance, living habits, growth tendency, and other aspects are closely related to spirals. In particular, spiral rules are more complex and diverse in the animal kingdom. For example, hawks approach their prey in a spiral path; insects approach a light source in a spiral orbit. There are various spirals on animal horns and on snail shells, which are the products of the gradual succession of new and old structures.

There are spiral phenomena not only on creatures on Earth but also in the celestial motion of the galaxy. The Milky Way is a super large spiral structure, the center of which is the densest star gathering, known as the "galactic core". The surrounding stars rotate in the same direction around the silver core, known as the "silver disk" in China. The rotation of the silver core drives the rotations of the silver disk, which extends from the inside to the outside, forming the spectacular scene known as the galaxy [2]. Due to its attractive aesthetics, there are many studies dedicated to spirals. With the develop of computer graphics, there also are many interesting methods used to create aesthetic spiral patterns automatically; see [3-9].

Why is nature so predisposed to spirals? It is believed that this is due to the continuous optimization of structures no matter what their scale during the process of evolution. For instance, the spiral seed arrangement of sunflowers not only makes it hold more seeds but also makes the seed disk stronger. Therefore, sunflowers with their spiral symmetry would gradually dominate in the survival of the fittest $[10,11]$.

The hyperbolic geometry founded by Gauss, Bolyai, and Lobachevsky at the end of the 19th century broke the monopoly of Euclidean geometry. It solved the outstanding parallel postulate problem for two thousand years, which caused a profound revolution in the concept of geometry and space. Looking back on history, we clearly see that hyperbolic geometry has become the prelude and preparation for the emergence of relativity in the 20 th century $[12,13]$. Nonetheless, a notable problem is that the study on spiral structures of 
hyperbolic characteristic is extremely limited; people only defined several simple equations of hyperbolic spirals on Poincaré disc; see some examples shown in Figure 1 [14,15]. In fact, as an important geometric space, the structure of hyperbolic tilings is well studied; see some hyperbolic tilings and Escher-like hyperbolic patterns shown in Figures 2 and 3 [16-18]. However, it seems that no one has attempted to visualize spiral tilings with hyperbolic features [19]. Due to the attractive aesthetics of spirals and hyperbolic geometry, it is natural to consider the visualization of hyperbolic spiral patterns. The purpose of this paper is to fill this gap.

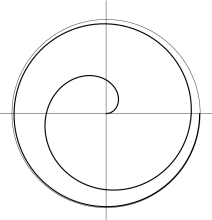

(a)

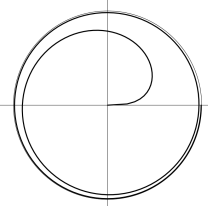

(b)

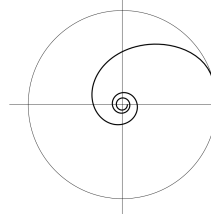

(c)

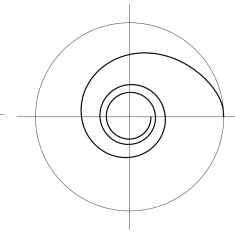

(d)

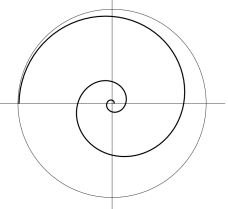

(e)

Figure 1. (a) Hyperbolic Archimedean spiral. (b) Hyperbolic Fermat spiral. (c) Hyperbolic reciprocal spiral. (d) Hyperbolic lotus spiral. (e) Hyperbolic golden spiral.

In this paper, we explore the method of visualizing hyperbolic spiral patterns. The rest of this paper is organized as follows. In Section 2, we propose a simple method to construct Euclidean spiral tilings of cyclic symmetry. In Section 3, based on a one-to-one correspondence between Euclidean plane and Poincaré disk as well as a conformal mapping between Poincaré and upper-half models, we first present two ways to construct hyperbolic spiral tilings. Then, to obtain Escher-like hyperbolic spiral patterns, we use wallpaper templates to render such hyperbolic spiral tilings. Finally, we conclude the paper in Section 4.

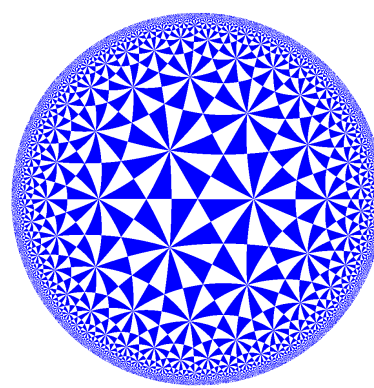

(a)

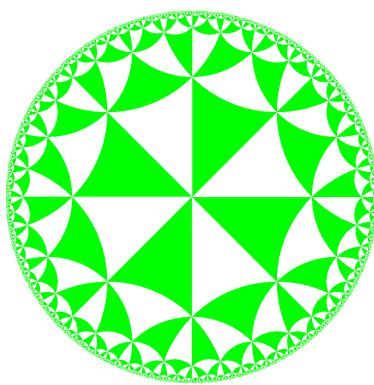

(b)

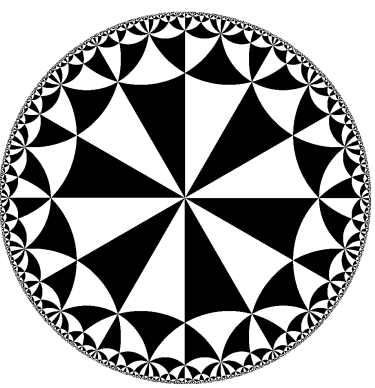

(c)

Figure 2. (a) A hyperbolic tiling of $\mathrm{T}(3,7,2)$ symmetry. (b) A hyperbolic tiling of $\mathrm{T}(4,4,4)$ symmetry. (c) A hyperbolic tiling of $\mathrm{T}(6,4,3)$ symmetry.

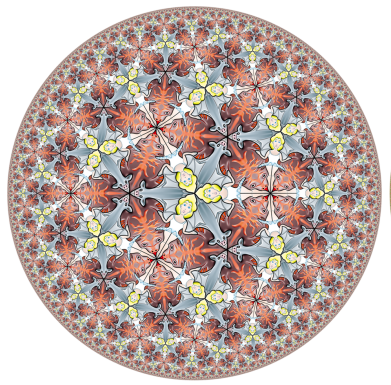

(a)

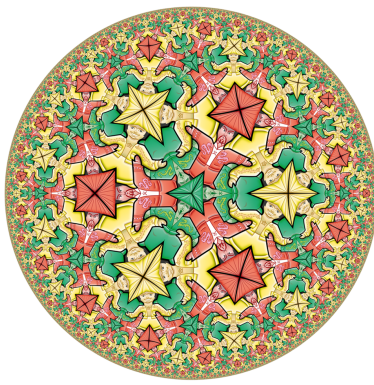

(b)

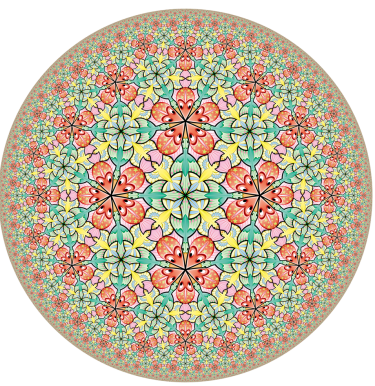

(c)

Figure 3. (a) An Escher-like Boar-girl-bat pattern of $\mathrm{T}(3,4,3)$ symmetry. (b) An Escher-like man pattern of $\mathrm{T}(3,4,4)$ symmetry. (c) An Escher-like pattern of $\mathrm{T}(4,5,4)$ symmetry. 


\section{Construction of Euclidean Spiral Tilings with Cyclic Symmetry}

In this section, we consider the construction of spiral tilings with cyclic symmetry in detail. It should be pointed out that the work carried out in this paper is an extension of [20]. We extend the results of the discussion in Euclidean space to hyperbolic space. Everything discussed in this section is similarly discussed in [20] (including Algorithm 1). However, for the sake of completeness and ease of reading, we summarize it again in a concise and easy-to-understand manner.

A logarithmic spiral is a curve in which the angle between pole diameter and its tangent is a constant. In this section, based on the logarithmic spiral, we consider the construction of cyclic spiral tilings in the Euclidean plane from the point of view of a symmetric group. We start by considering the equation associated with a logarithmic spiral in the polar coordinate system.

Let $\rho=\rho(\theta)$ be the polar curve in which the slope between the tangent and pole diameter is $k=\cot (\tau)$. That is, $\tau$ is the angle between pole diameter and its tangent. Then, we have

$$
\cos \tau \frac{\rho^{\prime}(\theta)}{\sqrt{\rho^{\prime 2}(\theta)+\rho^{2}(\theta)}}
$$

and

$$
\sin \tau=\frac{\rho(\theta)}{\sqrt{\rho^{\prime 2}(\theta)+\rho^{2}(\theta)}}
$$

by which we obtain

$$
\cot \tau=\frac{\rho^{\prime}(\theta)}{\rho(\theta)} .
$$

Rearranging the integral of (2), we finally obtain

$$
\rho(\theta)=c_{0} e^{\theta \cot \tau}, \theta \in \mathbb{R}, \text { where constant } c_{0} \in \mathbb{R} .
$$

To construct the spiral tilings of cyclic symmetry, we adopt a concise form of (3) as

$$
\rho(\theta)=e^{k \theta} .
$$

We next introduce some terms of symmetry groups and tilings. The symmetry of a tiling is a congruent or an isometric transformation. A tiling's symmetry group comprises all its symmetries. A set of generators with respect to $G$ is a subset of $G$ in which products comprise entire symmetries of $G$. The fundamental region $\Omega$ associated with $G$ is a region in which transformed copies under the action of $G$ just cover the entire plane, without overlapping and interstices. Geometrically, we can use $\Omega$ to tile the whole plane and to obtain a tiling with $G$ symmetry $[19,21]$.

Assume $i$ is the square root of -1 (i.e., $i=\sqrt{-1}$ ). Denote $\mathbb{R}^{+}, \mathbb{Z}^{+}$, and $\mathbb{C}$ as sets of positive real numbers, positive integers, and complex numbers, respectively. Consider a group $\Psi_{n}^{m}$ generated by

$$
g_{1}(z)=e^{\frac{2 \pi}{n} i} z
$$

and

$$
g_{2}(z)=e^{-\frac{2 \pi}{m}(k+i)} z
$$

where $k \in \mathbb{R}^{+}, n, m \in \mathbb{Z}^{+}$, and $z \in \mathbb{C}$. According to the theory of complex analysis, obviously, $g_{1}$ is equivalent to a counterclockwise rotation of $\frac{2 \pi}{n}$ about the origin. The geometrical meaning of $g_{2}$ is a bit complex. It is equivalent to a reduced transformation of $e^{-\frac{2 \pi}{n} \cot \tau}$ first and, then, follows a clockwise rotation of $\frac{2 \pi}{n}$ about the origin. Consequently, $g_{2}$ is a clockwise spiral contraction. 
Let $\Omega$ be a region bounded by lines

$$
\begin{gathered}
\zeta_{1}(\theta)=\frac{2 \pi}{m}, \\
\zeta_{2}(\theta)=0
\end{gathered}
$$

and spiral arcs

$$
\begin{gathered}
\omega_{1}(\theta)=e^{k \theta} \text { with } \theta \in\left[0, \frac{2 \pi}{m}\right], \\
\omega_{2}(\theta)=e^{k \theta} \text { with } \theta \in\left[\frac{2 \pi}{n}, \frac{2 \pi}{n}+\frac{2 \pi}{m}\right] .
\end{gathered}
$$

Equivalently, $\Omega$ can be represented as

$$
\Omega=\left\{z\left|0 \leq \arg (z) \leq \frac{2 \pi}{m}, e^{\arg (z)} \leq\right| z \mid \leq e^{\arg (z)+\frac{2 \pi}{n}}\right\},
$$

where $\arg (z) \in[0,2 \pi)$ is the main argument of $z$. We next investigate the action of $\Omega$ under symmetry group $\Psi_{n}^{m}=<g_{1}, g_{2}>$. Let $\rho_{1}(\theta)$ be the counterclockwise rotation of $\frac{2 \pi}{n}$ of the spiral $\rho(\theta)$, i.e.,

$$
\rho_{1}(\theta)=e^{k \theta+\frac{2 \pi}{n}} .
$$

Let $\Pi$ be a spiral arm-like region bounded by $\rho_{1}(\theta)$ and $\rho(\theta)$. More precisely,

$$
\Pi=\left\{z\left|e^{2 j \pi} \leq\right| z \mid e^{-\arg (z)} \leq e^{\frac{2 \pi}{n}+2 j \pi}, j=0, \pm 1, \pm 2, \pm 3, \cdots\right\} .
$$

On the other hand, $\Pi$ can be also regarded as an arm-like tiling resulting from $g_{2}$ and $\Omega$, i.e.,

$$
\Pi=\bigsqcup_{k=-\infty}^{+\infty} g_{2}^{k}(\Omega)
$$

According to the geometrically meaning of $g_{1}$, we have

$$
\mathbb{C}=\bigsqcup_{p=1}^{n} \bigsqcup_{k=-\infty}^{\infty} g_{1}^{p}\left(g_{2}^{k}(\Omega)\right)
$$

In other words, we can use tile $\Omega, g_{1}$ and $g_{2}$ to construct a spiral tiling. Thus, the $\Omega$ defined in (10) is a fundamental region associated with $\Psi_{n}^{m}$. Figure 4 shows the geometric sketch of $\Omega$.

By (14), in Figure 5, we show three cyclic spiral tilings on the Euclidean plane. For clarity, adjacent tiles in the spiral tiling are colored differently.

According to the geometrical meaning of $\Omega$, for $z \in \mathbb{C} \backslash \Omega$, there exists a certain equivalence point $z_{0} \in \Omega$, which can be realized by the following four steps:

1. Let $n_{1}=\left\lfloor\frac{\arg (z)}{\frac{2 \pi}{m}}\right\rfloor \in \mathbb{Z}^{+}$and $z_{1}=g_{2}^{-n_{1}}(z)$, where $\lfloor\chi\rfloor$ represents the maximum integer less than $\chi$. We have $0 \leq \arg \left(z_{1}\right) \leq \frac{2 \pi}{m}$.

2. Suppose $e^{2 n_{2} k \pi} \leq z_{1} \cdot e^{-\arg \left(z_{1}\right)(k+i)} \leq e^{2\left(n_{2}+1\right) k \pi}$ for certain $n_{2} \in \mathbb{Z}$ (note that $z_{1} \cdot e^{-\arg \left(z_{1}\right)(k+i)}$ is a real number). Let $n_{2}=\left\lfloor\frac{\ln \left|z_{1}\right|-k \arg \left(z_{1}\right)}{2 k \pi}\right\rfloor$ and $z_{2}=z_{1} \cdot e^{-\arg \left(z_{1}\right)(k+i)} \cdot e^{-2 n_{2} k \pi}$. Then, $z_{2} \in\left[1, e^{2 k \pi}\right]$ is a real number.

3. Let $n_{3}=\left\lfloor z_{2} \cdot e^{-\frac{2 k \pi}{n}}\right\rfloor \in \mathbb{Z}^{+}$and $z_{3}=z_{2} \cdot e^{-\frac{2 n_{3} k \pi}{n}}$. Then, $z_{3} \in\left[1, e^{\frac{2 k \pi}{n}}\right]$.

4. $z_{0}=z_{3} \cdot e^{\arg \left(z_{1}\right)(k+i)} \in \Omega$.

For future use, we call this algorithm Spiral Algorithm and summarize the corresponding pseudocode in Algorithm 1. 

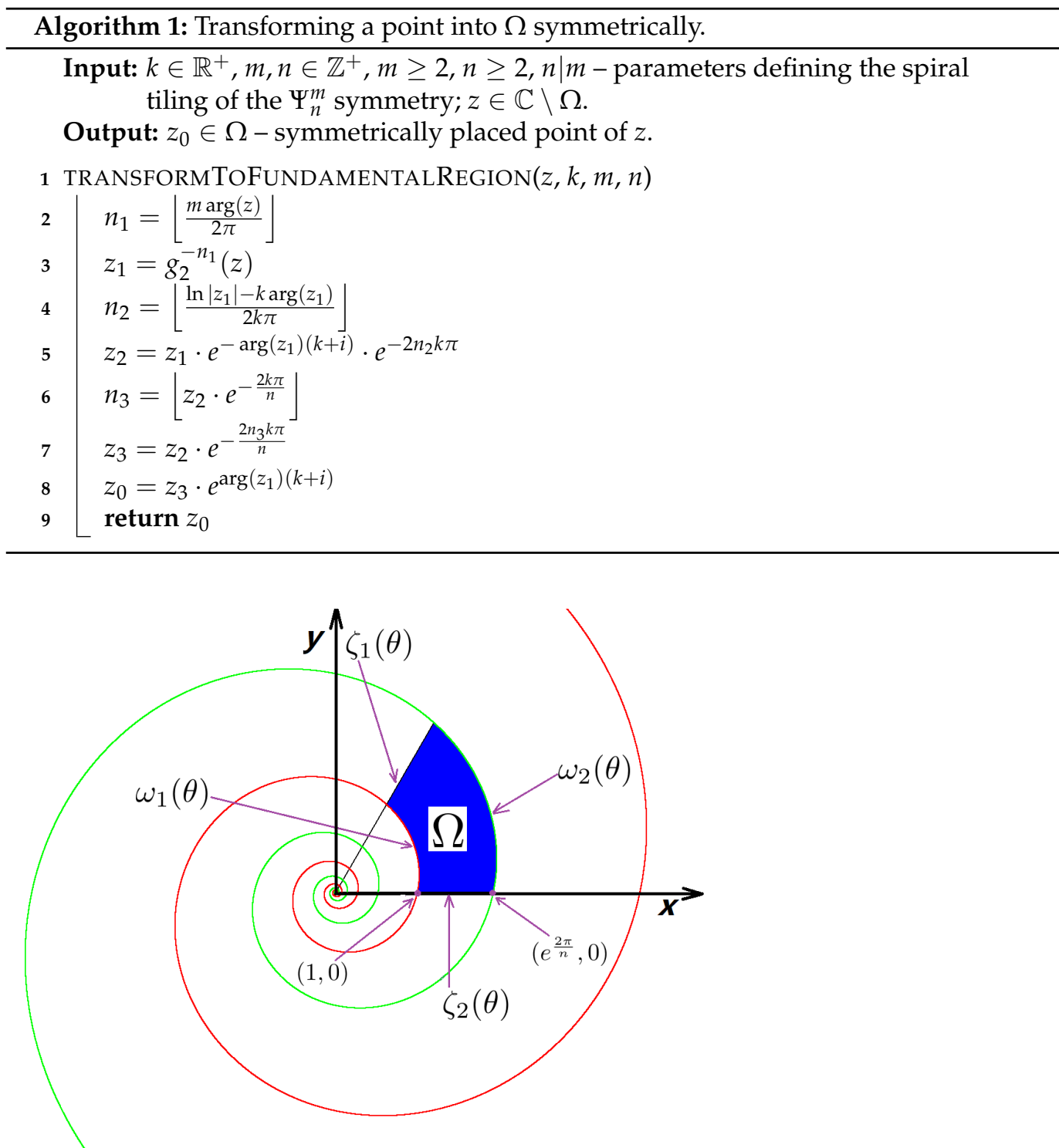

Figure 4. The blue region $\Omega$ enclosed by lines $\zeta_{1}$ and $\zeta_{2}$, and spiral arcs $\omega_{1}$ and $\omega_{2}$ is a fundamental region with respect to symmetry group $\Psi_{n}^{m}$.

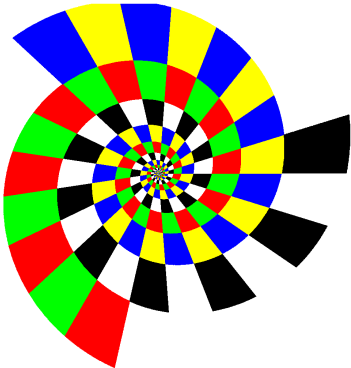

(a)

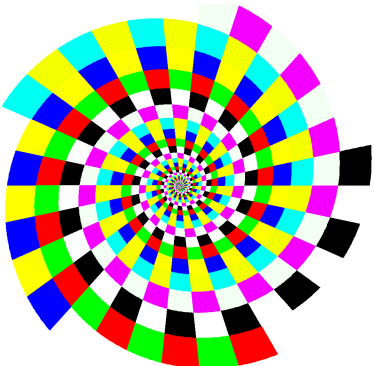

(b)

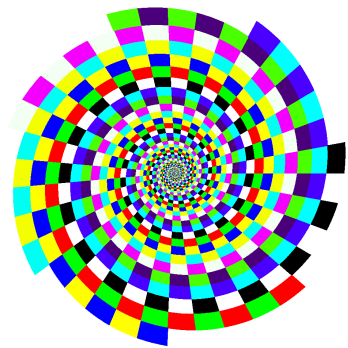

(c)

Figure 5. (a) A cyclic spiral tiling of $\Psi_{3}^{7}(k=0.2)$ symmetry. (b) A cyclic spiral tiling of $\Psi_{5}^{6}(k=0.15)$ symmetry. (c) A cyclic spiral tiling of $\Psi_{7}^{5}(k=0.12)$ symmetry.

\section{Hyperbolic Spiral Tilings and Patterns on Poincaré and Upper-Half Models}

With the preparations in Section 2, in this section, we first present a method to construct spiral tilings on hyperbolic space. Then, to produce Escher-like hyperbolic patterns, we use wallpaper templates to render hyperbolic spiral tilings. 
There are many different models for representing hyperbolic geometry in the Euclidian setting. In this paper, we restrict our discussion on Poincaré model

$$
\mathbb{D}=\{w|| w \mid<1, w \in \mathbb{C}\}
$$

and upper half-plane model

$$
\mathbb{H}^{2}=\{h \mid \operatorname{im}(h)>0, h \in \mathbb{C}\} .
$$

A mapping is conformal (angle preserving) at point $u$ if it preserves the angles oriented between curves through $u$ with respect to their orientation. Conformal mappings preserve the angles and shapes of infinitesimally small figures but not necessarily those patterns size. The conformal mapping between $\mathbb{D}$ and $\mathbb{H}^{2}$ is

$$
w=F(h)=\frac{1+h i}{h+1}, h \in \mathbb{H}^{2}, w \in \mathbb{D} .
$$

For $w \in \mathbb{D}$, the distance between $w$ and the origin of $\mathbb{D}$ can be defined as

$$
\frac{1}{2} \ln \frac{1+|w|}{1-|w|}
$$

Let $\partial \mathbb{D}$ be the boundary of $\mathbb{D}$. By (18), we see that $\partial \mathbb{D}$ is the infinity of Poincaré model. Consider a one-to-one correspondence between Euclidean plane and Poincaré disk as

$$
\begin{gathered}
z=M(w)=\frac{1}{2} \ln \frac{1+|w|}{1-|w|} e^{\arg (w)}, w \in \mathbb{D} . \\
\mathbb{H}=\left\{z \mid z=M(w)=\frac{1}{2} \ln \frac{1+|w|}{1-|w|} e^{\arg (w)}, w \in \mathbb{D}\right\} .
\end{gathered}
$$

Given $k \in \mathbb{R}^{+}, n, m \in \mathbb{Z}^{+}$, we summarize how to produce spiral tilings on Poincaré model $\mathbb{D}$. First, by (19), for $w \in \mathbb{D}$, we compute the corresponding point $z$; namely, this point ' $z$ ' is regarded as ' $z$ ' in Algorithm 1. Second, by the Spiral Algorithm established in Section 2, we use $g_{1}$ and $g_{2}$ to find the symmetrically placed point $z_{0} \in \Omega$ associated with $z$. According to this method, we can produce spiral tilings on Poincaré disc.

In Figure 6, we show six tilings obtained in this manner. We see that tiles near the origin of $\mathbb{D}$ have the similar structure as that in Figure 5. However, as tiles move toward to infinite boundary of $\mathbb{D}$, they become narrower and longer, and all of the tiles cannot cover entire Euclidean plane.

By the one-to-one conformal mapping (17) and the creation method of Figure 6, we can similarly produce hyperbolic spiral tilings on the upper half-plane model; see the four tilings shown in Figure 7. As this model is an infinite space, tilings in Figure 7 are displayed in a relatively interesting area $[-1.25,1.25][0.0001,1]$. The further away from the central area, the less obvious the spiral feature of the tilings.

By template, we mean the rectangle pattern decorated with recognisable motifs (such as animals or plants). It is well-known that wallpaper templates that have periodicity along the horizontal and vertical directions can be used to render spiral patterns $[20,22,23]$.

Assume $z_{0}$ is a point in the fundamental region $\Omega$ with respect to group $\Psi_{n}^{m}$. To produce Escher-like patterns, we need to color coordinates $(u, v)$ of $z_{0}$ correctly, which can be achieved by using argument and modulus of $z_{0}$. The $u$ coordinate is given by

$$
u=\frac{m \arg \left(z_{0}\right)}{2 \pi},
$$

while the formula for the $v$ coordinate is given by 


$$
v=\frac{\left|z_{0}\right|-1}{e^{\frac{2 \pi}{n}}-1}
$$

Figure 8 shows six Escher-like hyperbolic spiral patterns on a Poincaré disc. We see that, in the region near the origin of Poincare disc, those patterns have Euclidean spirals. Figure 9 shows four Escher-like hyperbolic spiral patterns on the upper half-plane. Although a spiral feature is not obvious in the upper half-plane model, the resulting Escher-like patterns are still visually appealing due to the intrinsic symmetries of hyperbolic geometry.

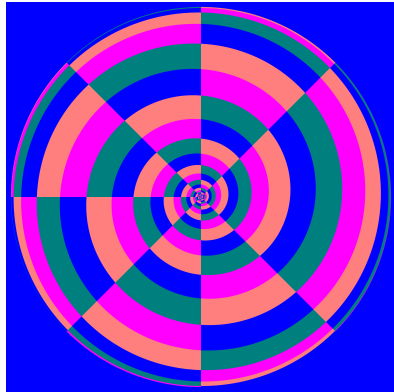

(a)

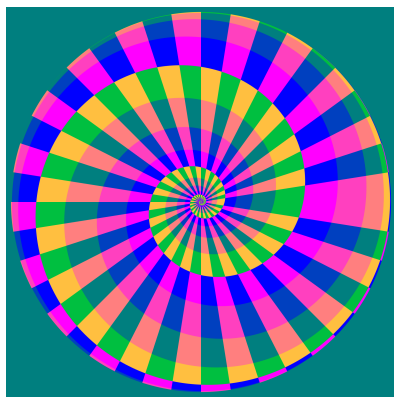

(d)

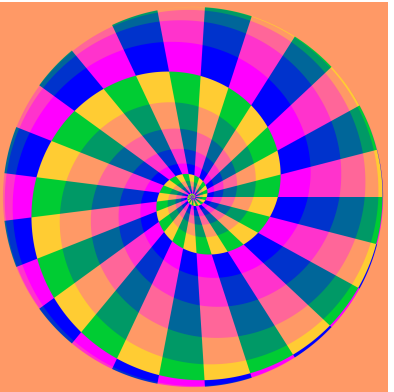

(b)

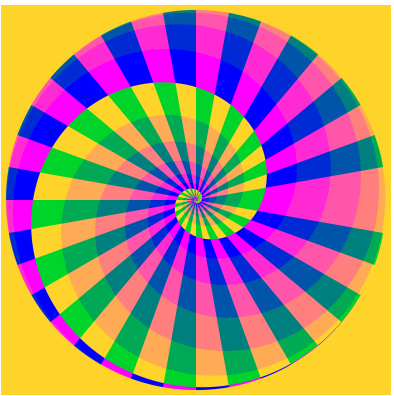

(e)

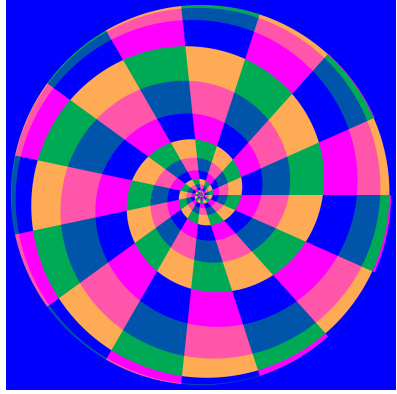

(c)

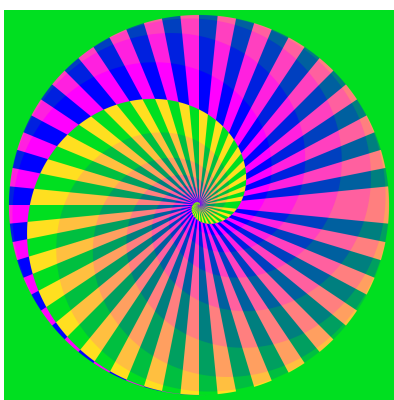

(f)

Figure 6. (a) Spiral tilings of $\Psi_{2}^{8}(k=0.1)$ symmetry on a Poincaré disc. (b) Spiral tilings of $\Psi_{2}^{13}$ $(k=0.3)$ symmetry on a Poincaré disc. (c) Spiral tilings of $\Psi_{3}^{5}(k=0.2)$ symmetry on a Poincaré disc. (d) Spiral tilings of $\Psi_{4}^{10}(k=0.25)$ symmetry on a Poincaré disc. (e) Spiral tilings of $\Psi_{6}^{6}(k=0.4)$ symmetry on a Poincaré disc. (f) Spiral tilings of $\Psi_{8}^{8}(k=0.25)$ symmetry on a Poincaré disc.

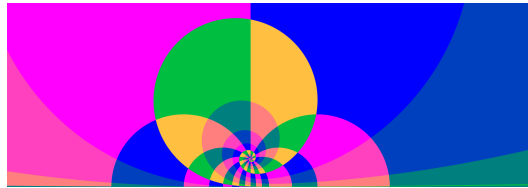

(a)

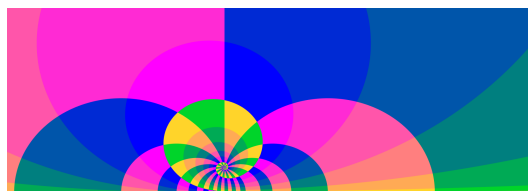

(c)

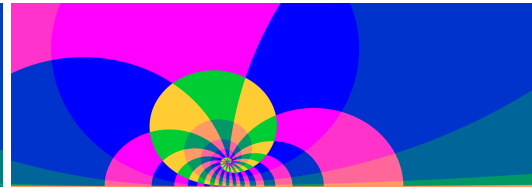

(b)

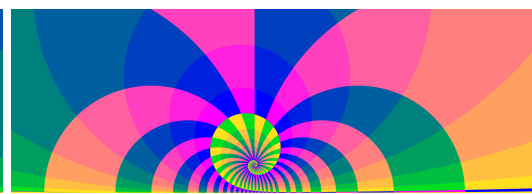

(d)

Figure 7. (a) Spiral tilings of $\Psi_{4}^{4}(k=0.3)$ symmetry on an upper half-plane. (b) Spiral tilings of $\Psi_{5}^{5}$ $(k=0.35)$ symmetry on an upper half-plane. (c) Spiral tilings of $\Psi_{6}^{4}(k=0.25)$ symmetry on an upper half-plane. (d) Spiral tilings of $\Psi_{8}^{6}(k=0.4)$ symmetry on an upper half-plane. 


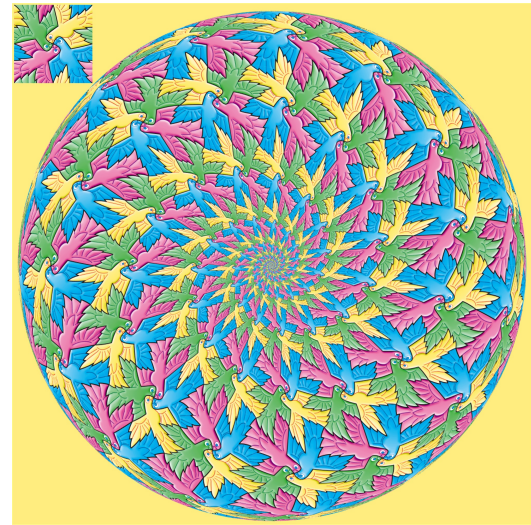

(a)

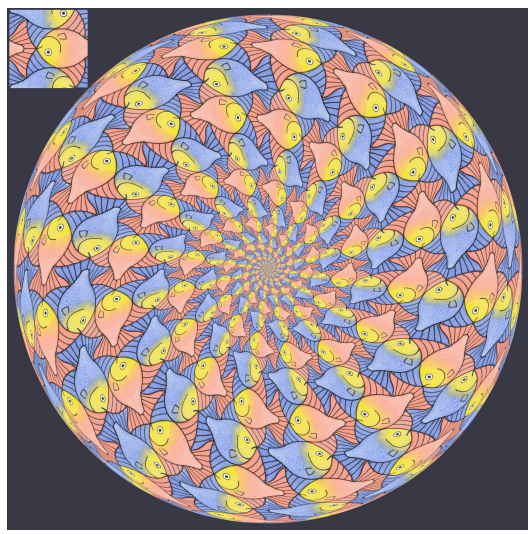

(c)

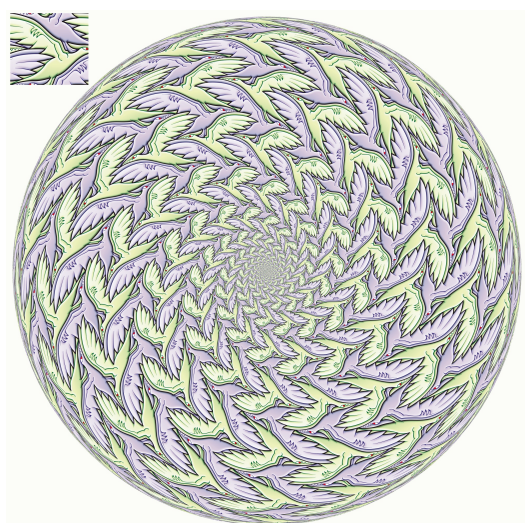

(e)

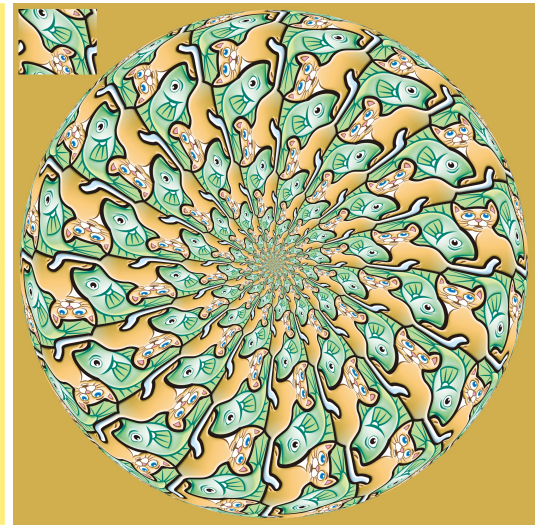

(b)

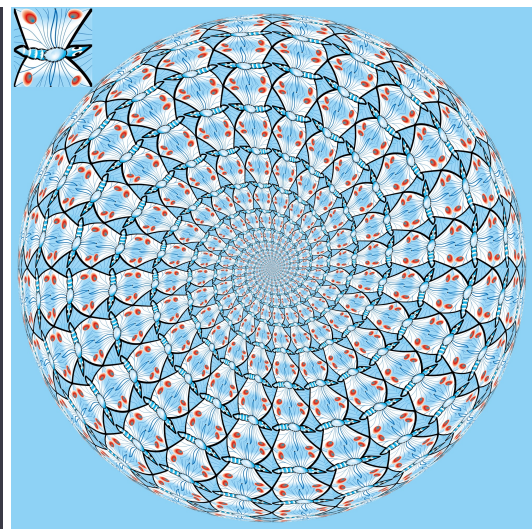

(d)

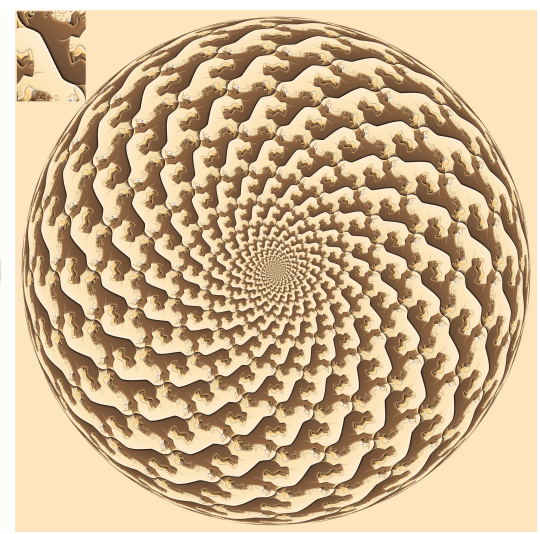

(f)

Figure 8. (a) An Escher-like bird pattern of $\Psi_{3}^{5}(k=0.35)$ symmetry on a Poincaré disc. (b) An Escherlike cat-fish pattern of $\Psi_{4}^{4}(k=0.5)$ symmetry on a Poincaré disc. (c) An Escher-like fish pattern of $\Psi_{4}^{4}(k=0.45)$ symmetry on a Poincaré disc. (d) An Escher-like butterfly pattern of $\Psi_{4}^{6}(k=0.25)$ symmetry on a Poincaré disc. (e) An Escher-like pterodactyl pattern of $\Psi_{6}^{3}(k=0.5)$ symmetry on a Poincaré disc. (f) An Escher-like dog pattern of $\Psi_{4}^{6}(k=0.255)$ symmetry on a Poincaré disc. The top left of each pattern is the wallpaper template rendered in the pattern. 


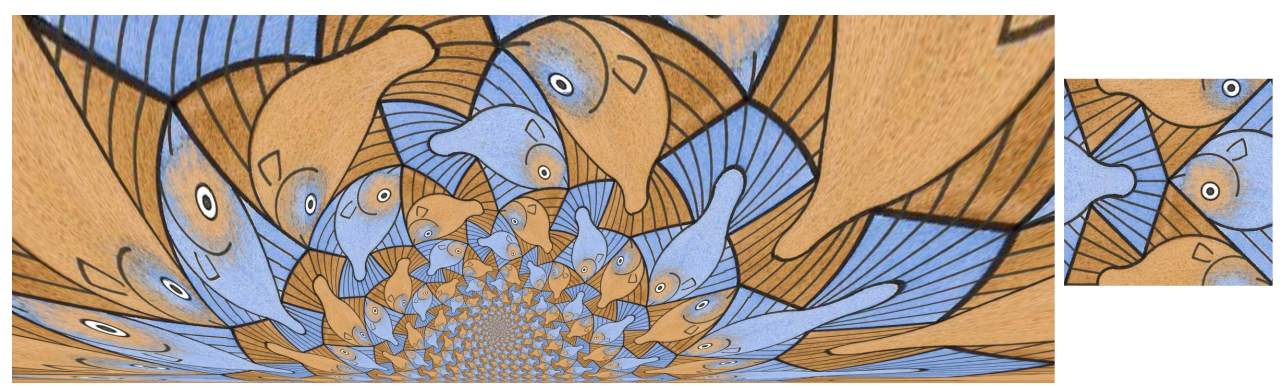

(a)

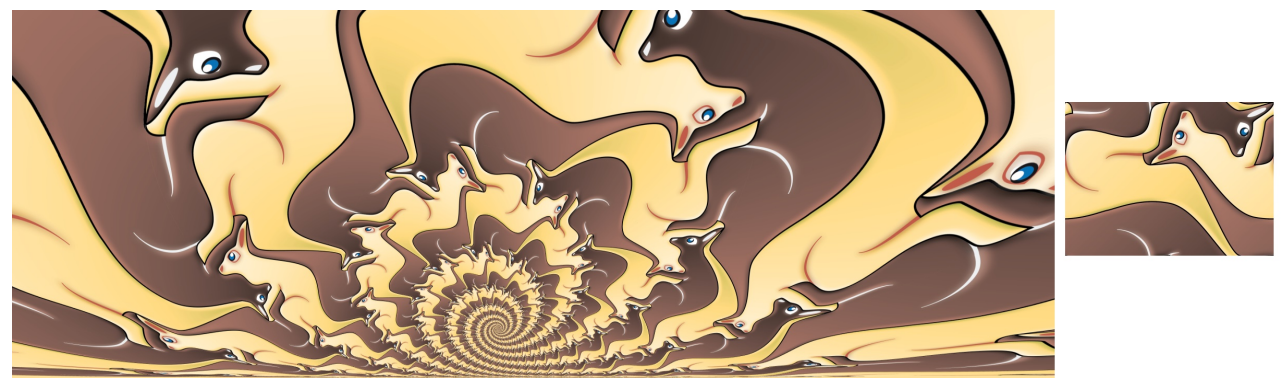

(b)
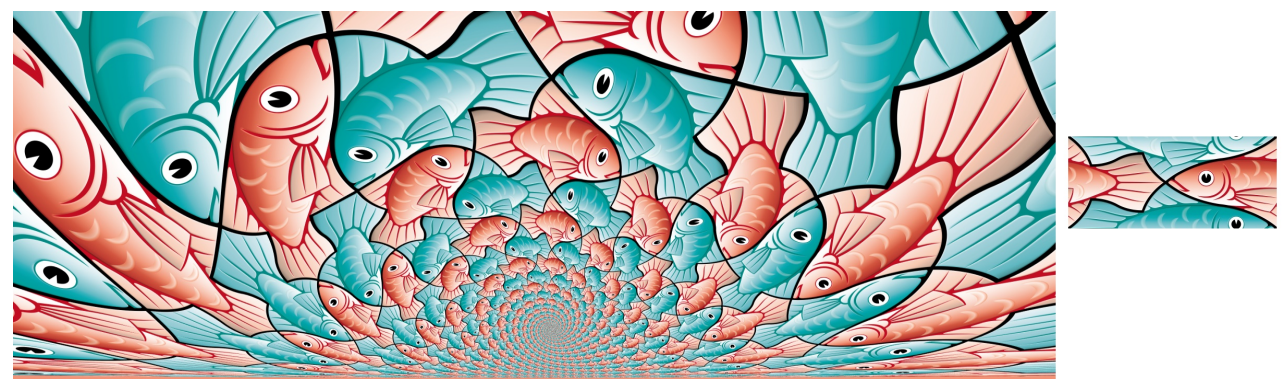

(c)
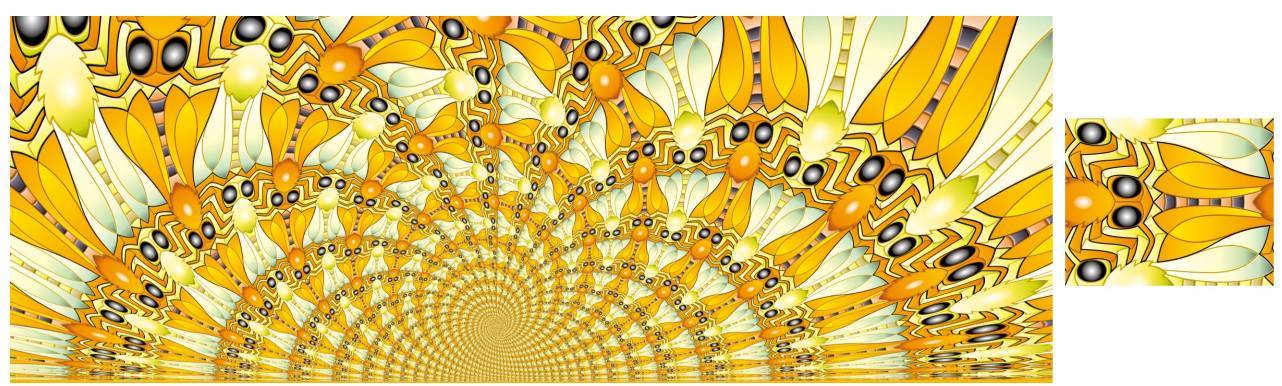

(d)

Figure 9. (a) An Escher-like fish pattern of $\Psi_{4}^{4}(k=0.3)$ symmetry on the upper half-plane. (b) An Escher-like kangaroo pattern of $\Psi_{5}^{5}(k=0.35)$ symmetry on the upper half-plane. (c) An Escher-like fish pattern of $\Psi_{6}^{4}(k=0.25)$ symmetry on the upper half-plane. (d) An Escher-like bee pattern of $\Psi_{8}^{6}$ $(k=0.4)$ symmetry on the upper half-plane. The right side of each pattern is the wallpaper template rendered in the pattern.

\section{Conclusions}

In this paper, we presented a simple method for visualizing hyperbolic spiral tilings and creating Escher-like patterns on tilings. To this end, we first introduced an approach for constructing spiral tilings in the Euclidean plane. Then, using the one-to-one correspondence between the Euclidean plane and the Poincaré disk, we introduce two ways to construct spiral tilings on the hyperbolic models. Finally, we specified the way to use wallpaper templates to render hyperbolic spiral tilings and to obtain Escher-like patterns. 
The method proposed is thus able to generate a great variety of Escher-like hyperbolic spiral patterns.

Author Contributions: Conception, programming and proofreading-C.Q.; analysis, writing, improvement and revision of the manuscript-X.L., J.P. and P.O. All authors discussed the results and contributed to the final manuscript. All authors have read and agreed to the published version of the manuscript.

Funding: This research was supported by the Natural Science Foundation of China (Nos. 11761039, 62062042), by the Science and Technology Project of the Education Department of Jiangxi Province of China (No. GJJ190542), by the Natural Science Foundation of Jiangxi Province of China (No. 20202ACBL211001), by the Science and Technology Project of Jiangxi Provincial Department of Education (No. 171495), by the Base and Talent Project of Guangxi Science and Technology (No. AA21196008), by the Guangxi Natural Science Foundation (No. 2018GXNSFBA281185), by the Doctor Start-up Foundation of Guangxi University of Science and Technology (Nos. 21Z48, 19Z21), by the Foundation for Young and Middle-Aged Teachers Basic Ability Improvement the Universities in Guangxi (No. 2021KY0362), and by the Undergraduate Teaching Reform Project of Guangxi Higher Education (No. 2021JGA224).

Institutional Review Board Statement: Not applicable.

Informed Consent Statement: Not applicable.

Data Availability Statement: The data used to support the findings of this study are available from the corresponding author upon request.

Acknowledgments: The authors thank the editors and anonymous reviewers for their detailed review, valuable comments, and constructive suggestions. The wallpaper templates used in Figures 8 and 9 as well as Figures A2-A4 in Appendix A are courtesy of Alain Nicolas (https:/ / en.tessellations-nicolas. com/ (accessed on 17 November 2021)) and David Bailey (http:/ / www.tess-elation.co.uk/new-hom (accessed on 17 November 2021)).

Conflicts of Interest: The authors declare that there are no conflict of interest.

\section{Appendix A. Some Less Appealing Hyperbolic Spiral Patterns}

In Section 3, we explored Poincaré and upper-half models. It is well-known that there are still many other hyperbolic models and interesting mappings. In practice, we attempted several other mappings. However, the resulting patterns are not as appealing as that in Figures 8 and 9. Nonetheless, we show three more mappings here as an inspiration so that the interested readers can attempt other similar cases.

Let

$$
\mathbb{K}=\{k|, k|<1, k \in \mathbb{C}\}
$$

be the hyperbolic Klein model. The conformal mapping between $\mathbb{D}$ and $\mathbb{K}$ is

$$
w=G(k)=\frac{k}{1+\sqrt{1-|k|^{2}}}, k \in \mathbb{K}, w \in \mathbb{D} .
$$

Let $\mathbb{G}$ be a strip region defined as

$$
\mathbb{G}=\{g \mid g \in \mathbb{C},-1<i m(g)<1\} .
$$

The conformal mapping between $\mathbb{D}$ and $\mathbb{G}$ is

$$
w=M(g)=\frac{e^{\frac{\pi}{2} g}-1}{e^{\frac{\pi}{2} g}+1}, g \in \mathbb{G}, w \in \mathbb{D} .
$$

Let $N$ be the circle inversion defined as

$$
w=N(z)= \begin{cases}\frac{1}{\bar{z}}, & \text { if }|z| \geq 1 \\ z, & \text { if }|z|<1\end{cases}
$$


where $z \in \mathbb{C}$ and $w \in \mathbb{D}$.

According to conformal mappings (A2), (A4), and (A5), by the same strategy as described in Section 3, we can similarly produce hyperbolic spiral tilings and patterns on the Klein, strip, and circle inversion models; see Figures A1-A4.

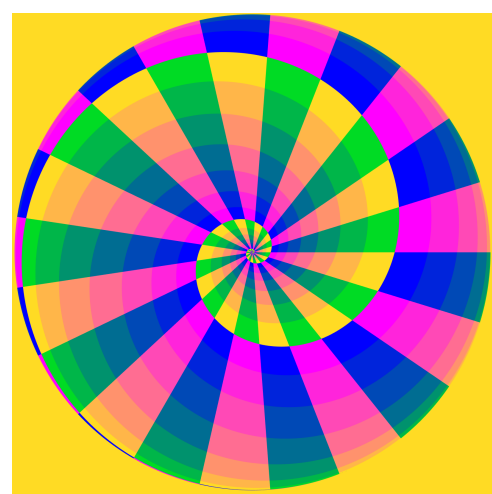

(a)

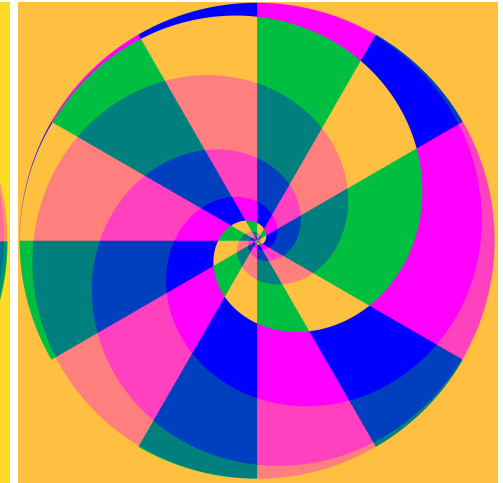

(b)

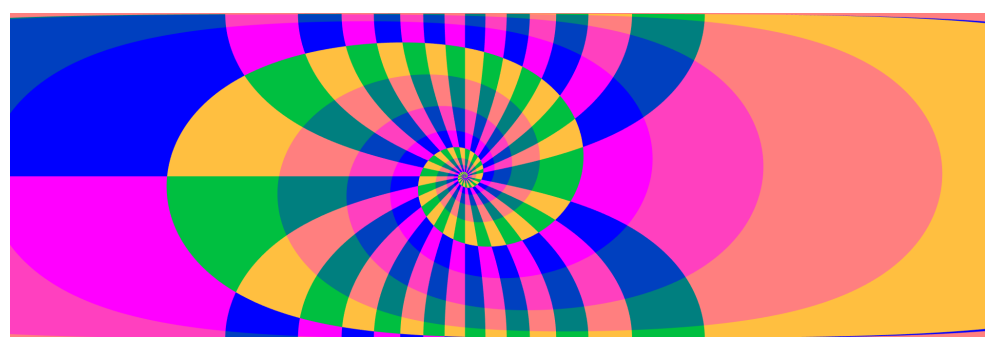

(c)

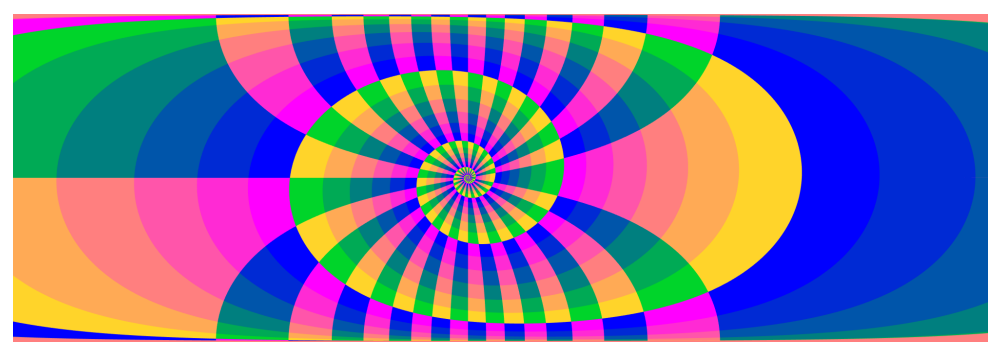

(d)

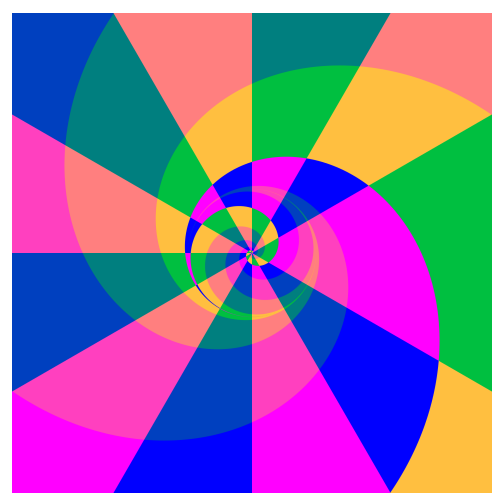

(e)

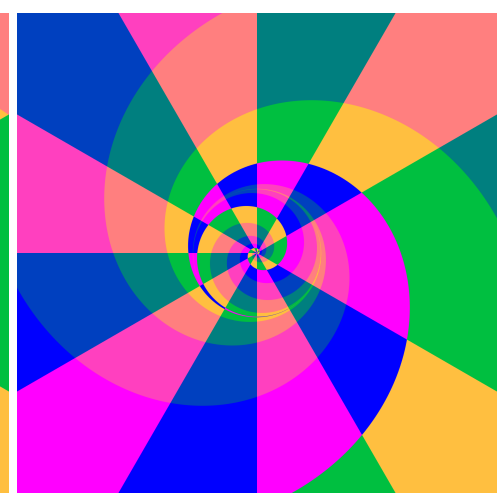

(f)

Figure A1. (a) Spiral tilings of $\Psi_{7}^{3}(k=0.5)$ symmetry on the Klein model. (b) Spiral tilings of $\Psi_{4}^{3}$ $(k=0.5)$ symmetry on the Klein model. (c) Spiral tilings of $\Psi_{4}^{8}(k=0.3)$ symmetry on the strip model. (d) Spiral tilings of $\Psi_{6}^{6}(k=0.2)$ symmetry on the strip model. (e) Spiral tilings of $\Psi_{4}^{3}(k=0.5)$ symmetry obtained by circle inversion. (f) Spiral tilings of $\Psi_{4}^{4}(k=0.4)$ symmetry obtained by circle inversion. 


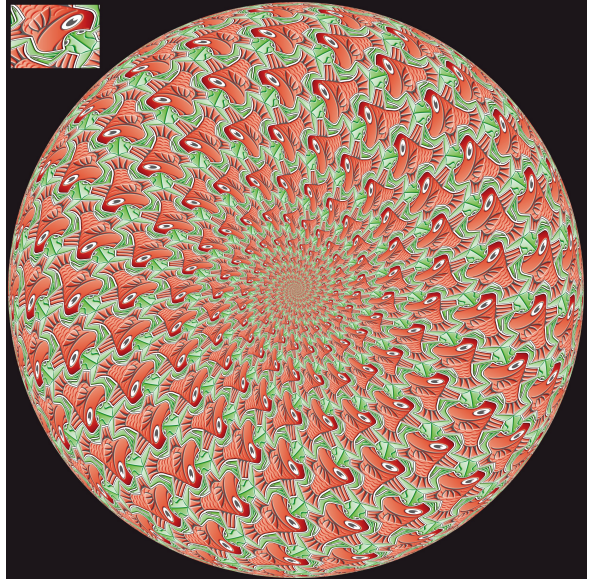

(a)

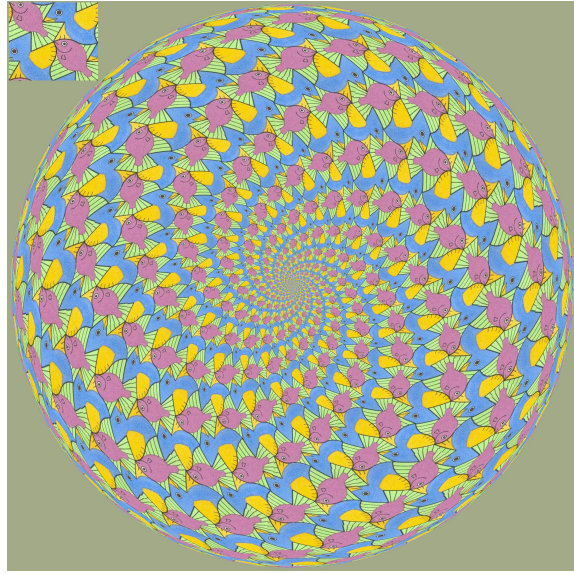

(c)

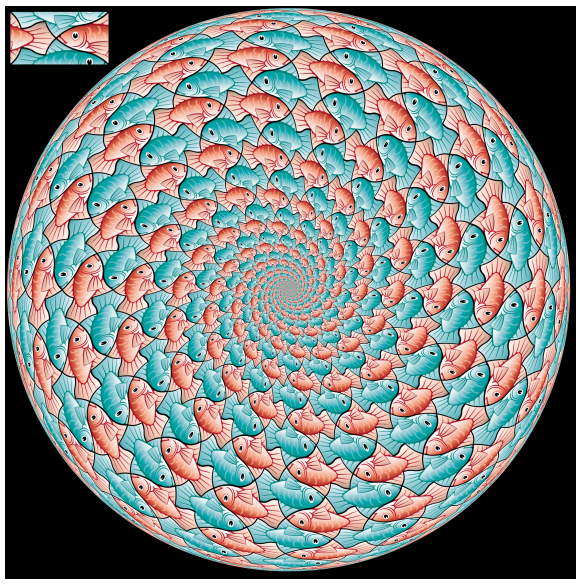

(b)

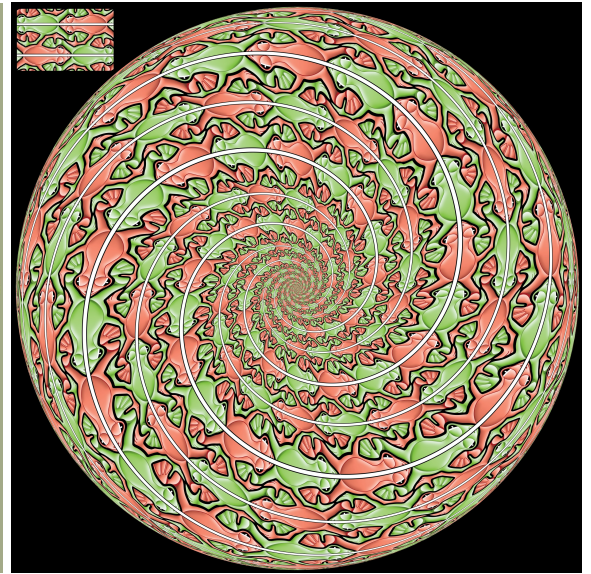

(d)

Figure A2. (a) An Escher-like frog-fish pattern of $\Psi_{5}^{5}(k=0.35)$ symmetry on the Klein model. (b) An Escher-like fish pattern of $\Psi_{6}^{6}(k=0.4)$ symmetry on the Klein model. (c) An Escher-like fish-bird pattern of $\Psi_{5}^{5}(k=0.4)$ symmetry on the Klein model. (d) An Escher-like frog pattern of $\Psi_{3}^{3}(k=0.2)$ symmetry on the Klein model. The top left of each pattern is the wallpaper template rendered in the pattern.

Although Klein and Poincaré have different metrics, the resulting Escher-like patterns in Figure A2 on the Klein model have similar structures as that in Figure 8 on the Poincaré model. It is not surprising since, the majority of the points in the unit disc mapping (A2) are approximately equal to a scale transformation. Thus, the patterns on the two models have no obvious difference.

For the Escher-like patterns in Figure A3 on the strip model, the space distorts too much and the resulting patterns are not visually appealing. As mapping (A5) fixes $\mathbb{D}$ and transforms points out of $\mathbb{D}$ into $\mathbb{D}$, for the Escher-like patterns in Figure $A 4$, the middle part of patterns has a disc structure similar to that in Figure 8 and Figure A2, while for the region out of the disc, there is an obvious Euclidean spiral structure. 


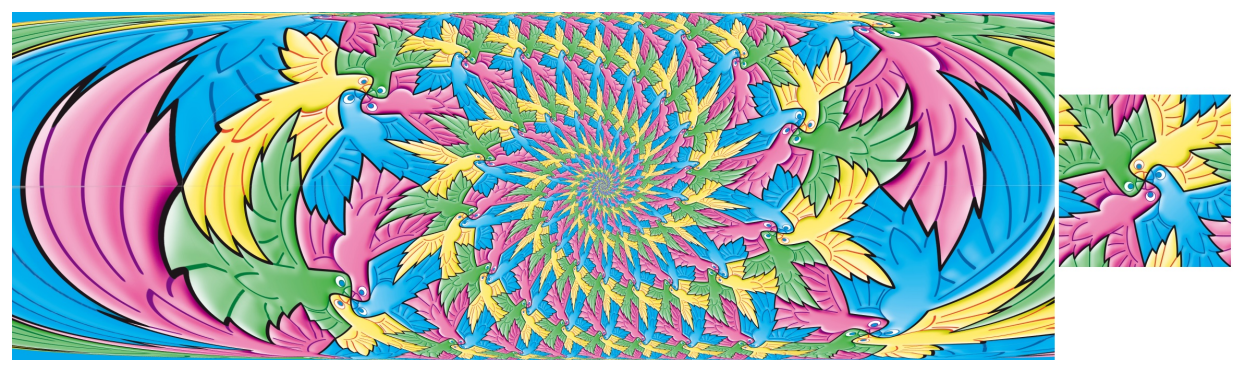

(a)

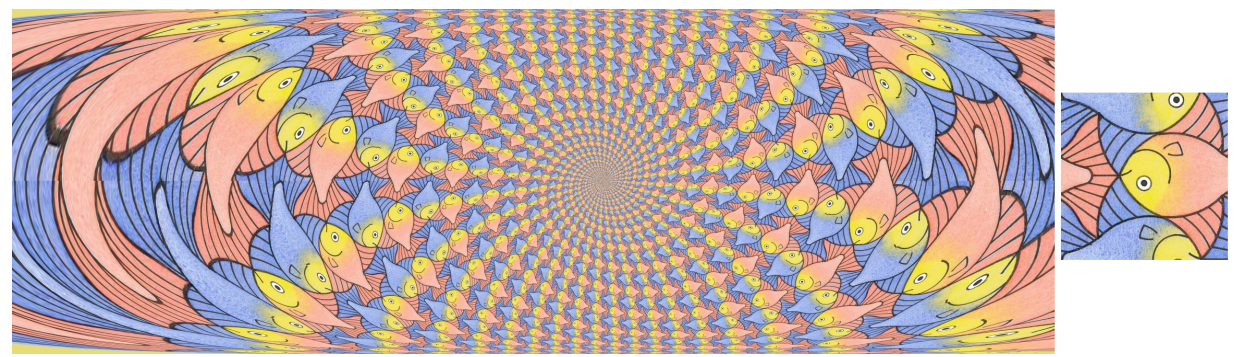

(b)

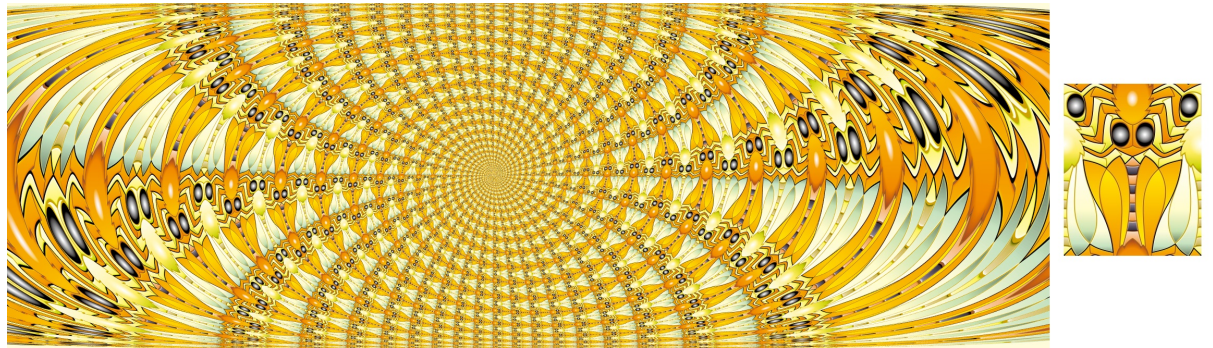

(c)

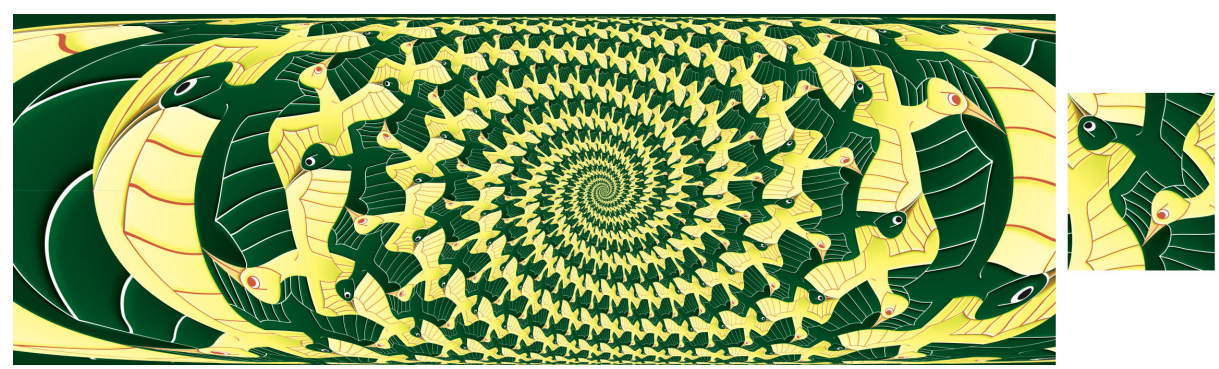

(d)

Figure A3. (a) An Escher-like bird pattern of $\Psi_{3}^{6}(k=0.4)$ symmetry on the strip model. (b) An Escher-like fish-bird pattern of $\Psi_{6}^{6}(k=0.3)$ symmetry on the strip model. (c) An Escher-like bee pattern of $\Psi_{6}^{6}(k=0.2)$ symmetry on the strip model. (d) An Escher-like bird pattern of $\Psi_{4}^{8}(k=0.3)$ symmetry on the strip model. The right side of each pattern is the wallpaper template rendered in the pattern. 


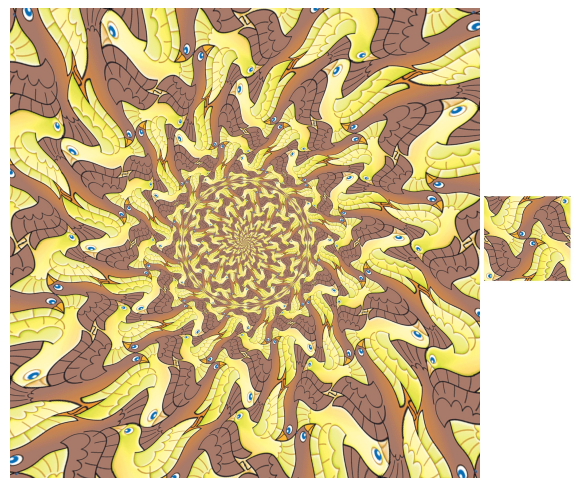

(a)

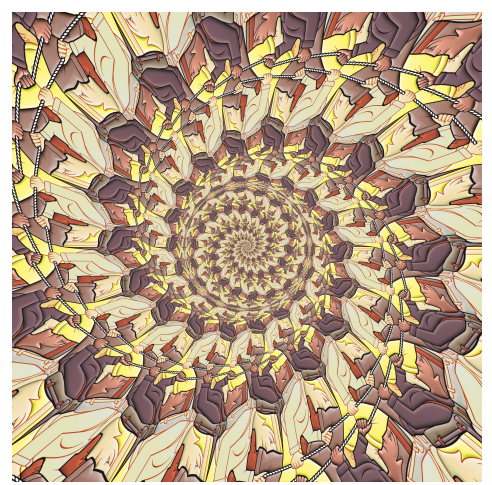

(c)

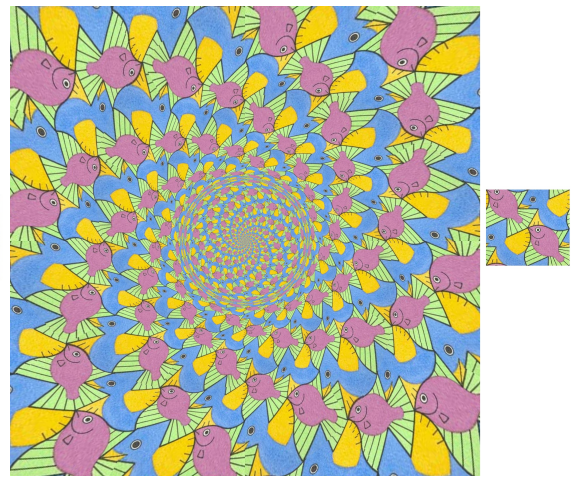

(b)

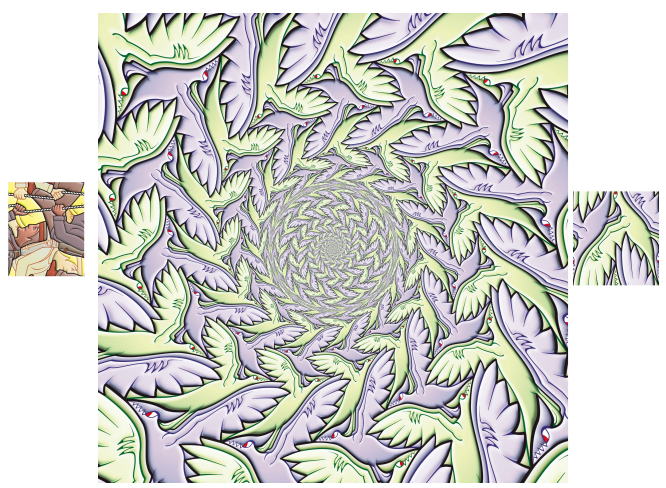

(d)

Figure A4. (a) An Escher-like bird pattern of $\Psi_{4}^{3}(k=0.5)$ symmetry obtained by circle inversion. (b) An Escher-like fish pattern of $\Psi_{4}^{3}(k=0.4)$ symmetry obtained by circle inversion. (c) An Escherlike man pattern of $\Psi_{3}^{6}(k=0.4)$ symmetry obtained by circle inversion. (d) An Escher-like pterodactyl pattern of $\Psi_{3}^{5}(k=0.35)$ symmetry obtained by circle inversion. The right side of each pattern is the wallpaper template rendered in the pattern.

\section{References}

1. Cook, T.A. The Curves of Life; University of Chicago Press: Chicago, IL, USA, 1994.

2. Hargittai, I.; Pickover, C. Spiral Symmetry; World Scientific: Singapore, 1992.

3. Chung, K.; Chan, H.; Wang, B. Spiral Tilings with Colour Symmetry from Dynamics. Comput. Graph. 1999, 23, 439-448. [CrossRef]

4. Ouyang, P.; Tang, X.; Chung, K.; Yu, T. Spiral Patterns of Color Symmetry from Dynamics. Nonlinear Dyn. 2018, 94, 261-272. [CrossRef]

5. Wang, X.; Ouyang, P. Beautiful Math: Aesthetic Patterns Based on Logarithmic Spirals. IEEE Comput. Graph. Appl. 2013, 33, 21-23. [CrossRef] [PubMed]

6. Dunham, D.; Lindgren, J.; Witte, D. Creating repeating hyperbolic patterns. ACM SIGGRAPH Comput. Graph. 1981, 15, 215-223. [CrossRef]

7. Carter, N.; Grimes, S.; Reiter, C. Frieze and wallpaper chaotic attractors with a polar spin. Comput. Graph. 1998, 22, 765-779. [CrossRef]

8. Gdawiec, K.; Kotarski, W. Polynomiography for the polynomial infinity norm via Kalantari's formula and nonstandard iterations. Appl. Math. Comput. 2017, 307, 17-30. [CrossRef]

9. Gdawiec, K. Inversion Fractals and Iteration Processes in the Generation of Aesthetic Patterns. Comput. Graph. Forum 2017, 36, 35-45. [CrossRef]

10. Bertin, G.; Lin, C.C. Spiral Structure in Galaxies: A Density Wave Theory. Phys. Today 1996, 50, 66-68. [CrossRef]

11. Kamien, R.; Lubensky, T.; Nelson, P.; Hern, C. Direct determination of DNA twist-stretch coupling. Europhys. Lett. 1997, 38, 237-242. [CrossRef]

12. Milnor, W.J. Hyperbolic geometry: The first 150 years. Bull. Am. Math. Soc. 1982, 6, 9-25. [CrossRef]

13. Ungar, A.A. Einstein's velocity addition law and its hyperbolic geometry. Comput. Math. Appl. 2007, 53, 1228-1250. [CrossRef]

14. Fathauer, R.W. Fractal Patterns and Pseudo-tilings Based on Spirals. In Mathematical Connections in Art, Music and Science, Bridges. 2004. Available online: http:/ / archive.bridgesmathart.org/2004/bridges2004-203.html (accessed on 17 November 2021). 
15. Dunham, D. Hyperbolic Spirals and Spiral Patterns. In ISAMA-BRIDGES Conference Proceedings; Barrallo, J., Friedman, N., Maldonado, J., Martínez-Aroza, J., Sarhangi, R., Séquin, C., Eds.; University of Granada: Granada, Spain, 2003; pp. 521-528. Available online: http:/ / archive.bridgesmathart.org/2003/bridges2003-521.html (accessed on 17 November 2021).

16. Ouyang, P.; Fathauer, R.; Chung, K.; Wang, X. Automatic Generation of Hyperbolic Drawings. Appl. Math. Comput. 2019, 347, 653-663. [CrossRef]

17. von Gagern, M.; Richter-Gebert, J. Hyperbolization of Euclidean Ornaments. Electron. J. Comb. 2009, 16, 1-12. [CrossRef]

18. Chung, K.W.; Wang, B.N. Tessellations with symmetries of the triangle groups from dynamics. Int. J. Bifurc. Chaos 2003, 13, 3505-3518. [CrossRef]

19. Grünbaum, B.; Shephard, G. Tilings and Patterns, 2nd ed.; Dover Publications: New York, NY, USA, 2016.

20. Ouyang, P.; Chung, K.W.; Bailey, D.; Nicolas, A.; Gdawiec, K. Generation of advanced Escher-like spiral tessellations. Vis. Comput. 2021, 1-13. [CrossRef]

21. Armstrong, M. Groups and Symmetry; Springer: New York, NY, USA, 1988.

22. Leys, J. Escher-like Interlocking Spiral Drawings. 2021. Available online: http://www.josleys.com/show_gallery.php?galid=290 (accessed on 17 November 2021).

23. Kaplan, C. Escher-like Spiral Tilings. 2019. Available online: https://isohedral.ca/escher-like-spiral-tilings / (accessed on 17 November 2021). 